\title{
Rapid micromotor-based naked-eye immunoassay
}

\author{
Berta Esteban-Fernández de Ávila ${ }^{\mathrm{a}}$, Mingjiao Zhao ${ }^{\mathrm{a}, \mathrm{b}}$, Susana Campuzano ${ }^{\mathrm{c}}$, Francesco Ricci ${ }^{\mathrm{b}}$, \\ José M. Pingarrón ${ }^{\mathrm{c}}$, Marcello Mascini ${ }^{\mathrm{a}, \mathrm{c}, \mathrm{d}, *}$, Joseph Wang ${ }^{\mathrm{a}, * *}$ \\ a Department of Nanoengineering,, University of California, La Jolla, San Diego, CA 92093, United States \\ b Department of Chemistry, University of Rome, Tor Vergata, Via della Ricerca Scientifica, 00133 Rome, Italy \\ c Department of Analytical Chemistry, Complutense University of Madrid, E-28040 Madrid, Spain \\ d Faculty of Bioscience and Technology for Food, Agriculture and Environment, University of Teramo, 64100 Teramo, Italy
}

\section{A R T I C L E I N F O}

\section{Keywords:}

Cortisol

Real-time sensing

Micromotor

Antibody

Naked-eye detection

\begin{abstract}
A B S T R A C T
A dynamic micromotor-based immunoassay, exemplified by cortisol detection, based on the use of tubular micromotors functionalized with a specific antibody is described. The use of antibody-functionalized micromotors offers huge acceleration of both direct and competitive cortisol immunoassays, along with greatly enhanced sensitivity of direct and competitive immunoassays. The dramatically improved speed and sensitivity reflect the greatly increased likelihood of antibody-cortisol contacts and fluid mixing associated with the dynamic movement of these microtube motors and corresponding bubble generation that lead to a highly efficient and rapid recognition process. Rapid naked-eye detection of cortisol in the sample is achieved in connection to use of horseradish peroxidase (HRP) tag and $\mathrm{TMB} / \mathrm{H}_{2} \mathrm{O}_{2}$ system. Key parameters of the competitive immunoassay (e.g., incubation time and reaction volume) were optimized. This fast visual micromotor-based sensing approach enables "on the move" specific detection of the target cortisol down to $0.1 \mu \mathrm{g} \mathrm{mL} \mathrm{m}^{-1}$ in just $2 \mathrm{~min}$, using ultrasmall $(50 \mu \mathrm{L})$ sample volumes.
\end{abstract}

\section{Introduction}

Recent advances in nanomotors have paved the way to novel biosensing systems and applications. Particularly, the ability of nano/ microscale motors to capture and transport specific target analytes in complex biological matrixes has been extensively explored [1-4].

Nano/micromotors functionalized with specific bioreceptors have thus allowed "on-the-fly" detection of different bioanalytes, ranging from nucleic acids to tumor cells [5-9]. Tubular micromotors functionalized with specific antibodies have been incorporated into lab-on-achip diagnostic devices demonstrating efficient recognition and isolation of the model protein IgG $[10,11]$. Such direct "on-the-fly" recognition and isolation processes obviate the need for common sampling and washing procedures. The efficient movement of these micromotors and generated-microbubbles tail has been shown to enhance the fluid mixing of the sample and to greatly increase the analyte-receptor interactions toward faster and more sensitive assays [12-14]. These dynamic sensing nanosystems have demonstrated important advantages to enhance target-bioreceptor interactions, which hold considerable interest for its implementation as medical diagnostic tools.
In this communication, we report on a new nanomotor immunoassay strategy based on naked-eye detection system. Cortisol, an important stress and clinical biomarker [15-19], was selected as model target to demonstrate the capabilities of this new approach. The most widely used methods in clinical assays of cortisol include luminescence and fluorescence assays [20,21], enzyme-linked immunosorbent assays (ELISAs) [22], and immunoassays in connection to fluorescence [23] or electrochemical detection [17,24-28]. However, these cortisol-detection methods still present some limitations in terms of analysis time, pre-treatment steps, and cost and do not involve rapid naked-eye detection.

The new dynamic cortisol detection strategy, described in this paper, is based on the use of tubular micromotors functionalized with a specific anti-cortisol antibody (Fig. 1). The advantages of such micromotor-based cortisol naked-eye detection system have been illustrated using direct and competitive immunoassay formats. As illustrated in Fig. 1, the direct motor-based immunoassay relies on HRP-labeled cortisol. The anti-cortisol functionalized-micromotors efficiently swim through the sample and detect rapidly and specifically the taggedcortisol target (Fig. 1A). The competitive motor assay involves competition of free-cortisol and the cortisol-HRP for the antibody sites on the

\footnotetext{
* Corresponding author at: Department of Nanoengineering, University of California, La Jolla, San Diego, CA 92093, United States.

** Corresponding author.

E-mail addresses: mmascini@unite.it (M. Mascini), josephwang@ucsd.edu (J. Wang).
} 

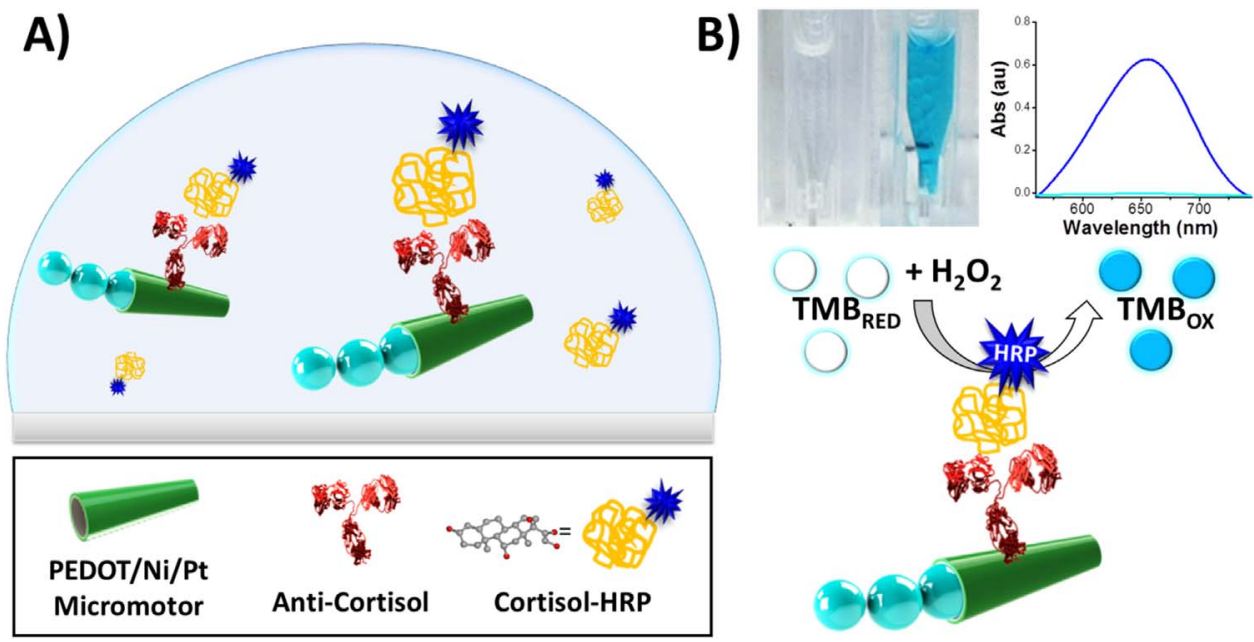

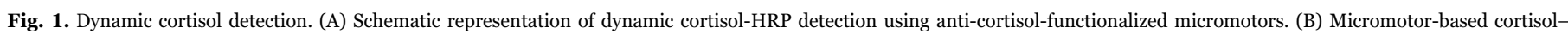

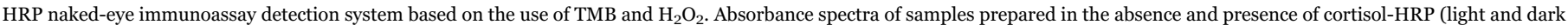

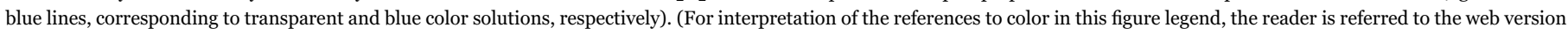
of this article).

moving micromotors. In both assays, short incubation of the resulting cortisol-HRP modified micromotors in a TMB $/ \mathrm{H}_{2} \mathrm{O}_{2}$ solution offers absorbance measurements of the deep blue product of TMB at $690 \mathrm{~nm}$ (Fig. 1B). The absorbance spectra of samples prepared in the absence and presence of cortisol-HRP (light and dark blue lines, corresponding to transparent and blue color solutions, respectively) are displayed in the top part of Fig. $1 \mathrm{~B}$.

The combination of the specific antibody with the efficient movement of the $\mathrm{H}_{2} \mathrm{O}_{2}$-propelled tubular micromotors allows rapid detection of dilute amounts of cortisol-HRP using microliter sample volumes. In comparison with early micromotor-based immunosensing strategies, involving direct or sandwich approaches and immunoreagents tagged with microsphere tracers for visualizing the antibodyantigen interaction $[10,11]$, this micromotor strategy demonstrated for the first time the use of a direct and competitive immunoassays with rapid naked-eye detection of the target analyte. As will be illustrated in the following sections, the movement of the antibody-modified micromotors through the sample plays a critical role in the enhanced biosensing performance. Such continuous motion of multiple anticortisol-micromotors imparts effective sample mixing within microliter samples that addresses the limitations associated with the slow cortisol transport under quiescent conditions and leads to fast antibodycortisol interactions and hence to higher speed and sensitivity.

\section{Materials and methods}

\subsection{Reagents}

A Cyclopore polycarbonate (PC) membrane, containing $5 \mu \mathrm{m}$ diameter conical-shaped micropores (Catalog No 110613; Whatman, Maidstone, U.K.), was employed as the template. Hydrocortisone (H4001, MW 362.46 $\mathrm{g} \mathrm{mol}^{-1}$ ), Sodium dodecyl sulfate (SDS, MW $288.38 \mathrm{~g} \mathrm{~mol}^{-1}$ ), 3,4-ethylenedioxythiophene (EDOT), potassium nitrate $\left(\mathrm{KNO}_{3}\right)$, hydrogen peroxide $\left(\mathrm{H}_{2} \mathrm{O}_{2}\right), 11$-Mercaptoundecanoic acid (MUA), 6-Mercapto-1-hexanol ( $\mathrm{MCH})$, 4-morpholineethanesulfonic acid hemisodium salt (MES), 1-ethyl-3-[3-dimethylaminopropyl] carbodiimide hydrochloride (EDC), and N-hydroxylsulfosuccinimide (Sulfo-NHS) were purchased from Sigma-Aldrich ${ }^{\circ}$. The monoclonal cortisol antibody (Catalog \# P01-92-94 M-P) and cortisol-HRP antigen (Catalog \# P91-92-91H) were purchased from EastCoast Bio ${ }^{\circledast}$. KBlue $^{\circledast}$ Aqueous 3,3',5,5'-tetramethylbenzidine (TMB) substrate was purchased from Neogen ${ }^{\circledR}$ Corporation.
The antibody was dissolved in $0.05 \mathrm{M}$ phosphate buffer (PBS) $\mathrm{pH}$ 7.4. The washing solution consisted of $0.05 \mathrm{M}$ phosphate buffer $\mathrm{pH} 7.4$ containing 0.05\% Tween-20.

The aqueous hydrogen peroxide solutions were used as the chemical fuel, and sodium dodecyl sulfate (SDS, MW $288.38 \mathrm{~g} \mathrm{~mol}^{-1}$ ) (at $3 \%$ final concentration) was used as a surfactant in all propulsion experiments.

Ultrapure water (18MX cm, Millipore Corporation, USA) was used for the preparation of all aqueous solutions.

\subsection{Apparatus}

Template electrochemical deposition of multi-layer microtubes was carried out with a CHI 661D potentiostat ( $\mathrm{CH}$ Instruments, Austin, TX). Scanning electron microscopy (SEM) images were obtained with a Phillips XL30 ESEM instrument, using an acceleration voltage of $20 \mathrm{kV}$. Energy-dispersive X-ray mapping analysis was performed using an Oxford EDX detector attached to SEM instrument and operated by INCA software. An inverted optical microscope (Nikon Instrument Inc. Ti-S/L100), coupled with a $20 \times$ and $40 \times$ objectives, a Photometrics QuantEM 512/SC camera (Roper Scientific, Duluth, GA) and MetaMorph 7.6 software (Molecular Devices, Sunnyvale, CA) were used for capturing movies at a frame rate of 30 frames per sec. The speed of the micromotors was tracked using a Metamorph tracking module and the results were statistically analyzed using Origin software.

The absorbance was measured between 500 and $800 \mathrm{~nm}$ using a UV-2450 Shimadzu spectrophotometer.

\subsection{Preparation of tubular micromotors}

The tubular micromotors were prepared using a common templatedirected electrodeposition protocol. A Cyclopore polycarbonate membrane, containing $5 \mu \mathrm{m}$ diameter conical-shaped micropores (Catalog No 110613; Whatman, Maidstone, U.K.), was employed as the template. A $75 \mathrm{~nm}$-thick gold film was first sputtered on one side of the porous membrane to serve as the working electrode using the Denton Discovery 18 sputtering system. The coating was performed at room temperature under base vacuum of $5 \times 10^{-6}$ Torr, DC power $200 \mathrm{~W}$ and $\mathrm{Ar}$ pressure of $3.1 \mathrm{mT}$, along with a rotation speed of $65 \mathrm{rpm}$ and sputtering time of $90 \mathrm{~s}$. A Pt wire and $\mathrm{Ag} / \mathrm{AgCl}(3 \mathrm{M} \mathrm{KCl})$ served as counter and reference electrodes, respectively. The mem- 
brane was then assembled in the electrochemical plating cell with an aluminum foil serving as a contact. Poly(3,4-ethylenedioxythiophene) (PEDOT) microtubes were electropolymerized at $+0.80 \mathrm{~V}$ using a charge of $0.1 \mathrm{C}$ from a plating solution containing $15 \mathrm{mM}$ EDOT, $7.5 \mathrm{mM} \mathrm{KNO}_{3}$ and $100 \mathrm{mM}$ SDS; subsequently, $\mathrm{Ni}$ was deposited from a Ni plating solution containing $\mathrm{NiCl}_{2} 3 \cdot 6 \mathrm{H}_{2} \mathrm{O}\left(20 \mathrm{~g} \mathrm{~L}^{-1}\right), \mathrm{Ni}\left(\mathrm{H}_{2} \mathrm{NSO}_{3}\right)_{2}$. $4 \mathrm{H}_{2} \mathrm{O}\left(515 \mathrm{gL}^{-1}\right)$, and $\mathrm{H}_{3} \mathrm{BO}_{3}\left(20 \mathrm{gL}^{-1}\right)$ at $-1.3 \mathrm{~V}(v \mathrm{sg} / \mathrm{AgCl})$ for $4{ }^{\circ} \mathrm{C}$. The inner Pt layer was galvanostatically deposited at $-2 \mathrm{~mA}$ for $500 \mathrm{~s}$ from a commercial platinum plating solution (Platinum RTP; Technic Inc, Anaheim, CA). The sputtered gold layer was completely removed by hand polishing with alumina slurry $(3-4 \mu \mathrm{m})$. After that, the membrane was dissolved in methylene chloride for $30 \mathrm{~min}$ to completely release the microtubes. The micromotors were collected by centrifugation at $7000 \mathrm{rpm}$ for $3 \mathrm{~min}$ and washed with methylene chloride, followed by ethanol and ultrapure water $(18.2 \mathrm{M} \Omega \mathrm{cm})$, three times for each, with a 3 min centrifugation following each wash. The template preparation method resulted in decidedly reproducible micromotors. The micromotors were placed over a glass slide and were sputtered with a layer of Au (DC power $200 \mathrm{~W}$, Ar pressure of $2.9 \mathrm{mT}$, along with a rotation speed of $65 \mathrm{rpm}$ and sputtering time of $40 \mathrm{~s}$ ) for the following functionalization with thiols chemistry. Finally, the Ausputtered micromotors were released from the glass slide by softly mechanical scratching.

Before performing the experiments, the micromotors were placed in $1 \mathrm{~mL}$ of water. In order to estimate the number of micromotors in solution, a $0.5 \mu \mathrm{L}$ drop of the micromotor suspension was observed under the microscope, and different optical images were taken from all the drop area, estimating the number of micromotors by counting the units observed in the images, and obtaining a final number of $1.1 \times 10^{5}$ micromotors per $\mathrm{mL}$.

$\mathrm{Au}$-sputtered PEDOT/Ni microtubes were used for static control experiments, following the same fabrication protocol but without the electrodeposition of platinum, which is responsible of the decomposition of $\mathrm{H}_{2} \mathrm{O}_{2}$ and consequent bubble production from the tubular micromotor.

\subsection{Micromotor functionalization}

The outer $\mathrm{Au}$ layer of the tubular micromotors or the static microtubes was functionalized first with a mixed MUA/MCH alkanethiol monolayer. A solution mixture of $2.5 \mathrm{mM}$ MUA and $7.5 \mathrm{mM}$ $\mathrm{MCH}$ was prepared in ethanol. Aliquots of $100 \mu \mathrm{L}$ were taken from the micromotor or microtube suspensions (containing $1.1 \times 10^{4}$ units, approximately), and after separation of the supernatant with centrifugation, the micromotors/microtubes were incubated in the MUA/MCH solution overnight. After washing the micromotors or microtubes with water for $5 \mathrm{~min}$, they were transferred to an eppendorf vial containing a $100 \mu \mathrm{L}$ MES buffer (pH 6.5) solution containing the coupling agents EDC and Sulfo-NHS at 0.4 and $0.1 \mathrm{M}$, respectively, and were incubated for $35 \mathrm{~min}$. After washing with PBS buffer $\mathrm{pH} 7.4$ for $5 \mathrm{~min}$, the micromotors or microtubes were incubated with the anti-cortisol antibody $\left(100 \mu \mathrm{g} \mathrm{m}^{-1}\right)$ during $2 \mathrm{~h}$ at room temperature, followed by two 15 min washing steps with a PBS solution ( $\mathrm{pH}$ 7.4) containing 0.05 wt\% tween-20 and one washing step with a PBS solution ( $\mathrm{pH} 7.4$ ) to remove the excess of antibody from the solution. The micromotors or microtubes were centrifuged and kept dried at $4{ }^{\circ} \mathrm{C}$ until use. Although the magnetic properties of the micromotors (given by the nickel layer) were enough for allowing magnetic separation, the micromotors/ microtubes were centrifuged to avoid their loss and ensure their efficient recover. A schematic illustration of the protocol used for anti-cortisol covalent immobilization is shown in Fig. S1.

\subsection{Detection of attached cortisol-HRP}

The antibody-functionalized micromotors were mixed with a PBS (pH 7.4) solution containing $1 \% \mathrm{H}_{2} \mathrm{O}_{2}$ fuel, $3 \%$ SDS surfactant and 1:100 of the cortisol-HRP target (all these variables were kept constant in all the experiments). The mixture was allowed to react during different times ( $30 \mathrm{~s}, 1,2,5$ or $10 \mathrm{~min}$ ) and also prepared in different volumes $(25,50$, and $100 \mu \mathrm{L})$. Afterwards, the micromotors were washed two times with PBS solution ( $\mathrm{pH} 7.4)$ containing $0.05 \mathrm{wt} \%$ tween-20 and another time with PBS (pH 7.4) to remove the excess of

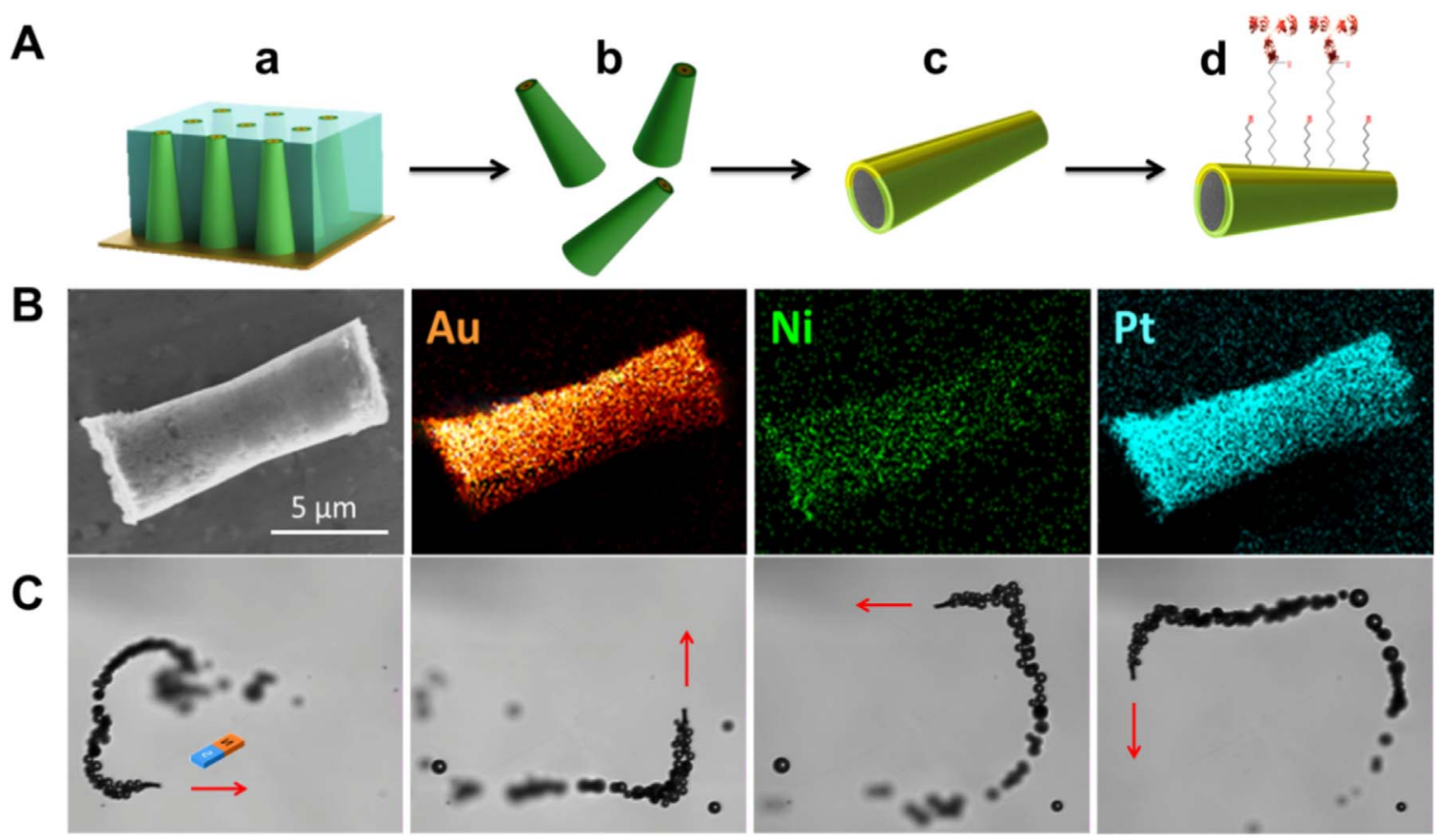

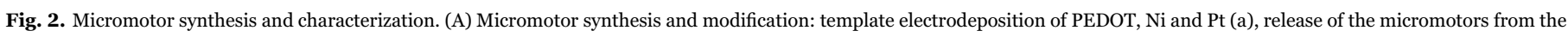

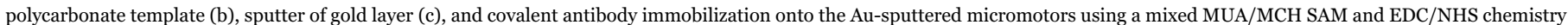

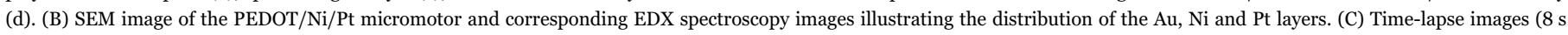

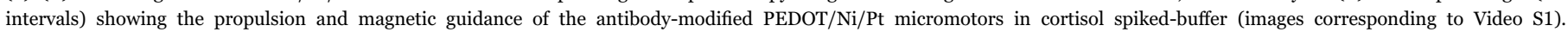
Experimental conditions: PBS pH 7.4 solution containing $1 \% \mathrm{H}_{2} \mathrm{O}_{2}, 3 \%$ SDS and 1:100 of cortisol-HRP. 

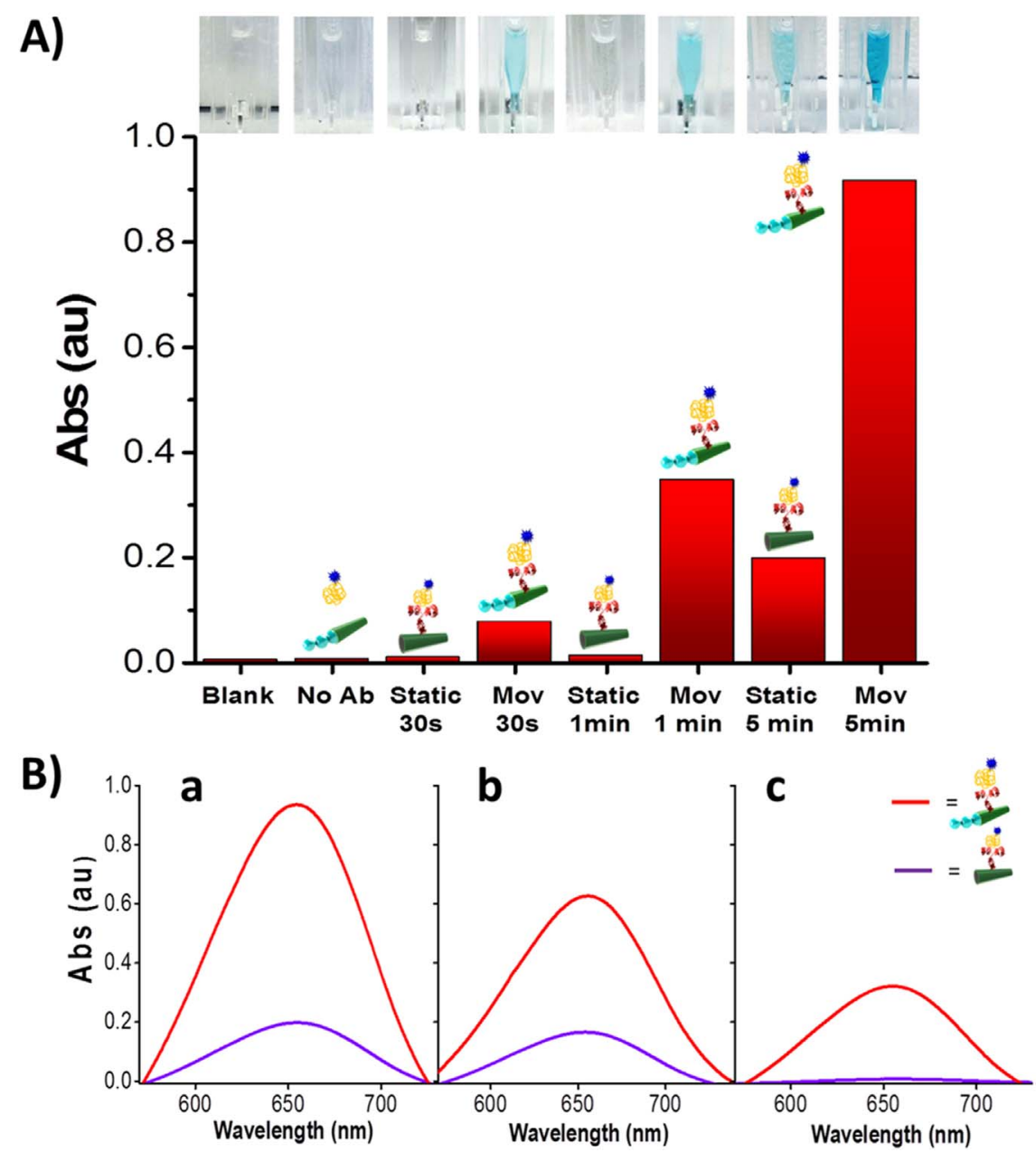

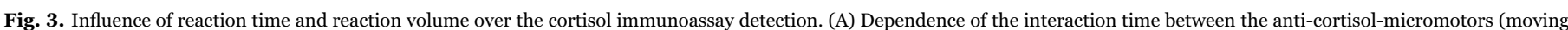

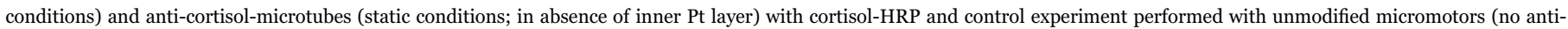

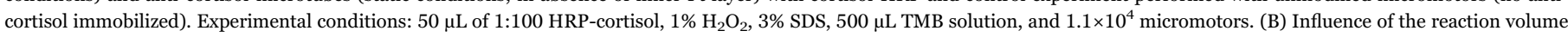

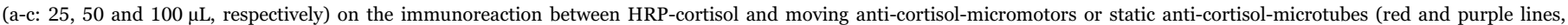

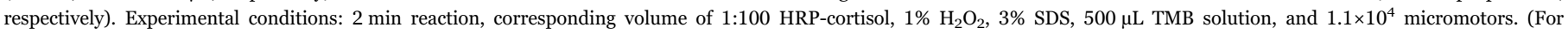
interpretation of the references to color in this figure legend, the reader is referred to the web version of this article).

unbounded cortisol-HRP. The cortisol-HRP-antibody-functionalized micromotors were separated by centrifugation and were mixed with $500 \mu \mathrm{L}$ of undiluted TMB solution (containing $\mathrm{H}_{2} \mathrm{O}_{2}$ ), and the absorbance spectra $(500-800 \mathrm{~nm})$ was measured. Notice that the TMB develops a deep blue color in the presence of $\mathrm{HRP}$ and $\mathrm{H}_{2} \mathrm{O}_{2}$. A schematic illustration of the protocol used for cortisol-HRP detection is shown in Fig. S1.

Since the micromotors used were propelled by the bubbles generated at the inner Pt surface and the $\mathrm{H}_{2} \mathrm{O}_{2}$ is required for TMB developing the blue color in the presence of HRP, anti-cortisolfunctionalized-Au-sputtered PEDOT/Ni microtubes (without Pt inner layer) were used to perform the static micromotors control experiments.

\subsection{Competitive immunoassay}

The direct competitive immunoassay for cortisol detection was performed by mixing $1.1 \times 10^{4}$ antibody-functionalized micromotors or microtubes (for static control experiments) with $50 \mu \mathrm{L}$ of a PBS (pH 7.4) solution containing $1 \% \mathrm{H}_{2} \mathrm{O}_{2}$ fuel, $3 \%$ SDS surfactant, different concentrations of a free cortisol standard $\left(0,0.1\right.$ and $\left.1 \mu \mathrm{g} \mathrm{mL} \mathrm{L}^{-1}\right)$ and $0.5 \mu \mathrm{L}$ of the commercial cortisol-HRP target solution (i.e., 1:100 dilution). After 2 and 10 min incubation when using micromotors and stationary microtubes, respectively, they were washed one time with PBS solution (pH 7.4) containing 0.05 wt\% tween-20 and another time with PBS ( $\mathrm{pH} 7.4$ ). Finally, the micromotors and microtubes were separated by centrifugation and were mixed with $500 \mu \mathrm{L} \mathrm{TMB} / \mathrm{H}_{2} \mathrm{O}_{2}$ undiluted commercial solution, and the corresponding absorbance spectra and value at $690 \mathrm{~nm}$ were measured.

\section{Results and discussion}

\subsection{Synthesis and characterization of micromotors}

Fig. 2A schematically illustrates the steps involved in the fabrication and functionalization of the multilayer PEDOT/Ni/Pt tubular micromotors with the anti-cortisol antibody. The micromotors used for detecting cortisol were fabricated by a standard membrane template electrodeposition method. Briefly, the template-electrodeposition protocol consisted on electropolymerization of the EDOT monomer within the conical micropores of a polycarbonate (PC) membrane used as a template, followed by electrodeposition of $\mathrm{Ni}$ and the inner Pt layer (Fig. 2A (a)), and release of the resulting PEDOT/Ni/Pt micromotors by dissolving the membrane (Fig. 2A (b)). The micromotors were subsequently sputtered with a thin layer of Au (Fig. 2A (c)) for further functionalization. The immobilization of the anti-cortisol antibody onto the outer Au layer of the micromotors was carried out using EDC/NHS chemistry through a mixed self-assembled monolayer (SAM) of MUA/ 

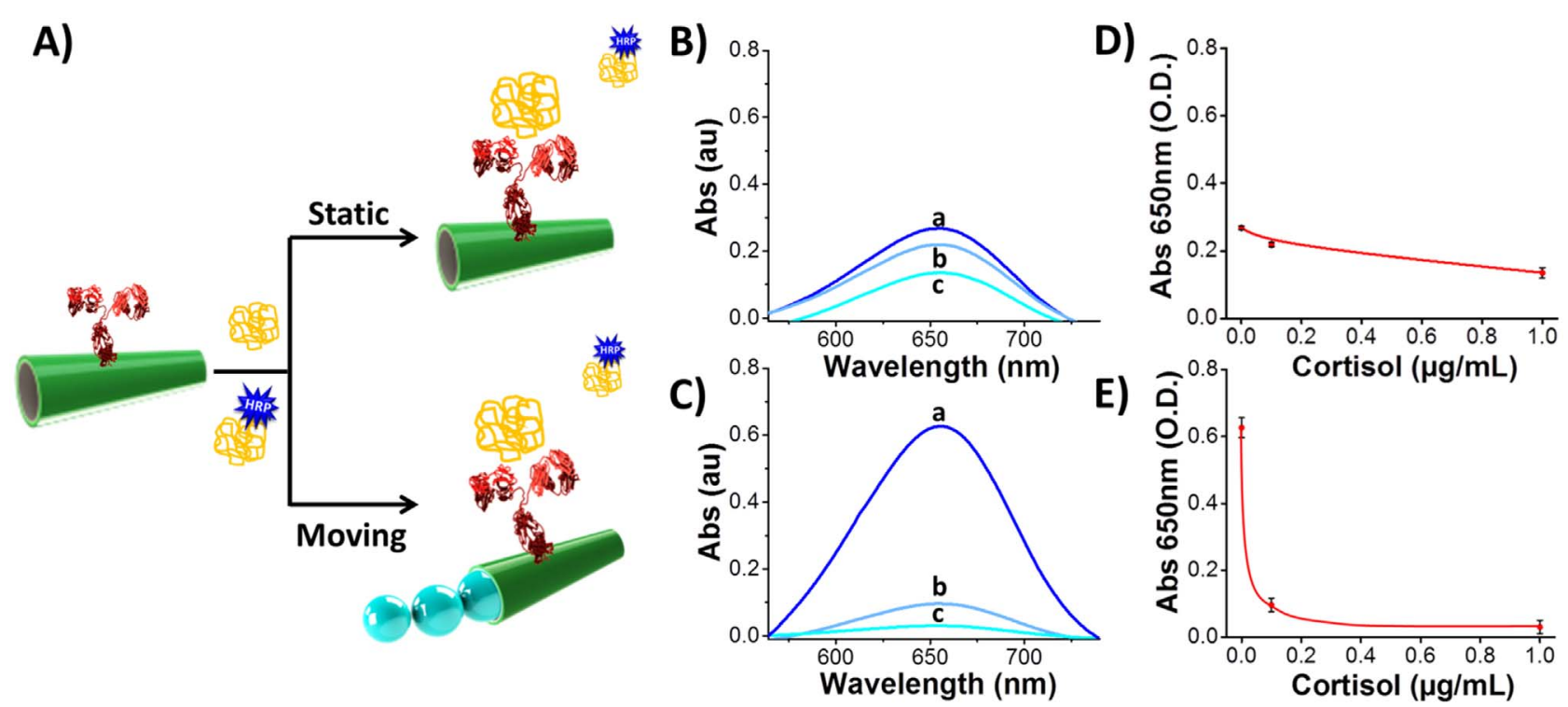

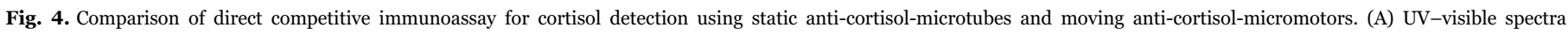

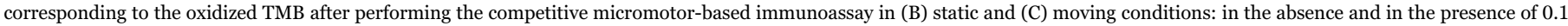

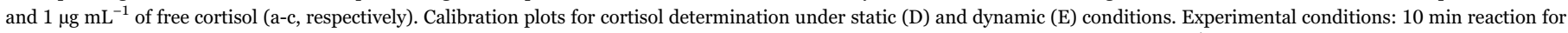
static microtubes and 2 min for moving micromotors, $50 \mu \mathrm{L}$ of $1: 100$ HRP-cortisol, $1 \% \mathrm{H}_{2} \mathrm{O}_{2}$, $3 \%$ SDS, $500 \mu \mathrm{L}$ TMB solution, and $1.1 \times 10^{4}$ micromotors.

$\mathrm{MCH}$ alkanethiols (Fig. 2A (d); detailed experimental protocol explained in Methods and in Fig. S1). As was mentioned above, Ausputtered PEDOT/Ni microtubes were used as static controls, following the same fabrication protocol but without the Pt electrodeposition, which is responsible for the catalytic $\mathrm{H}_{2} \mathrm{O}_{2}$ fuel reaction and consequent bubble production from the tubular micromotor.

Fig. 2B depicts the scanning electron microscopy (SEM) image of an Au-sputtered PEDOT/Ni/Pt micromotor with $\sim 10 \mu \mathrm{m}$ length and a $\sim 5 \mu \mathrm{m}$ diameter; the latter reflects the pore size of the PC membrane used in the fabrication process. Corresponding energy-dispersive X-ray (EDX) images of the Au-sputtered PEDOT/Ni/Pt micromotor confirmed the presence of the outer $\mathrm{Au}$ film, Ni intermediate layer, and an inner Pt layer (Fig. 2B), required for antibody functionalization, concentration and magnetic guidance, and catalytic propulsion in the presence of $\mathrm{H}_{2} \mathrm{O}_{2}$, respectively.

The propulsion of the antibody-functionalized Au-sputtered PEDOT/Ni/Pt micromotors was also studied. Time-lapse images, taken from Video S1, illustrate the propulsion of the antibody-functionalized micromotors in the presence of $1 \% \mathrm{H}_{2} \mathrm{O}_{2}$ fuel, along with the magnetic guidance of the micromotor though the cortisol spiked-buffer (Fig. 2C). Efficient movement at a speed of $80 \mu \mathrm{m} \mathrm{s}^{-1}$ is observed. The inclusion of the Ni layer in the composition of the micromotor facilitates the magnetic manipulation of the micromotors during the functionalization protocol, and opens the possibility of guiding these antibodyfunctionalized micromotors to the target destination.

\subsection{Optimization of detection conditions}

The development of the immunosensing nanomotor strategy has involved optimization of key experimental conditions, such as incubation time and reaction volume, and variety of relevant control experiments (Fig. 3).

Initial control experiments were performed by incubating anticortisol-micromotors in a blank solution (prepared in the absence of cortisol-HRP), or non-functionalized micromotors in a cortisol-HRP solution (Fig. 3A: bars 1 and 2, respectively). These resulted in negligible absorbance, thus indicating the absence of unspecific background contributions due to non-specific adsorptions of the enzymatic tracer onto the non-modified micromotors and discarding potential erroneous readings.
In order to demonstrate the important role of the self-propelled micromotors, and corresponding localized fluid convection, in the acceleration and efficiency of the cortisol binding and detection, the effect of the incubation time was investigated both under static and moving conditions, using anti-cortisol functionalized microtubes and micromotors, respectively. As shown in Fig. 3, a clear increase of the signal is observed when using dynamic anti-cortisol-micromotors compared to static anti-cortisol-microtubes. In particular, moving the micromotors for $30 \mathrm{~s}$ provided a 3-fold higher absorbance signal compared to static microtubes after 1 min incubation. This trend was confirmed by the double response provided by the dynamic micromotors at 1 min compared to that of the static microtubes after 5 min. Due to saturation of the absorbance above $5 \mathrm{~min}$, this was the longest time tested. These results also demonstrated that the antibodyfunctionalized micromotors could efficiently navigate in $1 \% \mathrm{H}_{2} \mathrm{O}_{2}$ supplemented media during at least $5 \mathrm{~min}$ without affecting their immunorecognition efficiency. Overall, the data of Fig. 3A confirm that the rapid movement of the anti-cortisol-micromotors through the sample greatly increases the likelihood of antibody-cortisol contacts, and thus leads to a highly efficient and rapid recognition process.

After studying the cortisol-detection ability of the anti-cortisolmicromotors at different incubation times, the effect of the reaction volume $(25,50$ and $100 \mu \mathrm{L})$ was investigated using a fixed reaction time of 2 min Fig. 3B demonstrates that the assay sensitivity increases substantially upon decreasing the sample volumes from 100 to $25 \mu \mathrm{L}$, reflecting the greatly enhanced likelihood of antibody-cortisol contacts using smaller sample volumes under these dynamic conditions. Overall, these preliminary experiments confirm that the use of dynamic micromotors clearly improves the antibody-cortisol interactions, to offer increasing sensitivity while reducing largely the assay time.

\subsection{Direct competitive assay}

In addition to direct immunoassays of cortisol, the micromotor approach can greatly enhance the performance of competitive cortisol immunoassays (Fig. 4).

These bioassays involve the competition of cortisol and HRP-tagged cortisol for the antibody sites on the moving micromotors (Fig. 4A). The direct competitive immunoassay format was carried out using both static and moving antibody-modified microtubes and micromotors to 
evaluate their recognition properties in a semi-quantitative manner. Using this approach, the quantity of cortisol-HRP, inversely proportional to the quantity of free cortisol in the sample, was rapidly estimated.

According to the results obtained from the previous optimization studies, the reaction volume selected for performing this competitive immunoassay was $50 \mu \mathrm{L}$, using reaction times of 2 and $10 \mathrm{~min}$ for dynamic and static conditions, respectively. The anti-cortisol functionalized static microtubes and micromotors were incubated in standard solutions prepared in the absence and in the presence of 0.1 and $1 \mu \mathrm{g} \mathrm{mL}^{-1}$ of free cortisol. As demonstrated results presented in Fig. 4B and $\mathrm{C}$, absorbance signals for cortisol-HRP detected under static and dynamic conditions decreased with increasing the free cortisol concentration, in agreement with the basis of a direct competitive immunoassay.

In order to evaluate the influence of the free cortisol binding efficiency upon the micromotors movement, competition plots obtained under moving and static conditions were compared. As it is shown in Fig. 4D and E, the moving micromotors offer a drastically rise in the negative slope of dynamic system response (Fig. 4E), nearly 5fold more than their static counterpart in the range $0-0.1 \mu \mathrm{g} \mathrm{mL}{ }^{-1}$ free cortisol concentration (Fig. 4D). In agreement with previous results obtained in the optimization of the incubation time, these data confirm the possibility of using these anti-cortisol functionalized micromotors to accelerate the selective antibody-target interaction while increasing the assay sensitivity and performance. These preliminary results demonstrate that the micromotor approach offers both the qualitative naked-eye detection of lateral flow assays (the most practical approaches to point of care cortisol testing) and the possibility to perform also quantitative analysis. The work has been focused on the comparison between the performances of dynamic micromotors and static microtubes. The narrow spectrophotometric range obtained using static microtubes did not allow to statistically quantifying concentrations below $0.1 \mu \mathrm{g} \mathrm{mL}^{-1}$. It is worth to mention at this point that the great influence of the number of motors, reaction volume and the incubation time on the immunoreaction efficiency allow tailoring the response range of this efficient micromotor-based immunoassay.

Overall, the presented results demonstrate that the combination of specific anti-cortisol antibodies with the efficient movement of selfpropelled micromotors allows rapid detection of dilute amounts of cortisol-HRP in microliter volume samples. The attractive capabilities demonstrated by these functionalized micromotors for rapid and effective cortisol sensing hold enormous potential for designing nextgeneration of miniaturized mobile diagnostic devices.

\section{Conclusions}

We have developed a very attractive micromotor-based immunosensing strategy for rapid, selective, single-step and naked-eye detection of cortisol. The new direct and competitive immunoassays exploit the transport properties of antibody-modified self-propelled micromotors for greatly enhanced speed and sensitivity in connection to an HRP tag for such naked-eye detection. Due to the increased localized fluid convection produced by their autonomous moving, the use of selfpropelled antibody functionalized micromotors offers efficient target binding using microliter samples, and results in dramatic acceleration compared to antibody functionalized static microtubes. Such naked-eye detection greatly simplifies the whole immunoassay protocol. The improved capabilities of this "on-the-flight" direct immunoassay allowed sensitive $\left(0.1 \mu \mathrm{g} \mathrm{L}^{-1}\right)$ and rapid $(2 \mathrm{~min})$ detection of cortisol in ultrasmall sample volumes. It is worth to mention also that the attractive capabilities offered by these moving micromotors are advantageous compared to that of commercial micromagnetic counterparts in bioassays implementation in terms of simplicity of operation and reaction time. The magnetic guidance, associated with its intermediate Ni layer, could facilitate movement through a microchannel network toward a cortisol microchip.
These results demonstrate that the dynamic cortisol detection strategy, based on the use of tubular micromotors functionalized with a specific anti-cortisol antibody, can offer significant improvements and facilitate high-performance to direct and competitive immunoassays of cortisol. These rapid micromotors cortisol assays could be used also for rapid screening purposes in connection to existing cortisol detection systems that demand more time and laborious protocols. While the new concept was presented in connection to the detection of cortisol, it may be readily translated to the detection of a broad range of target biomarkers, making these micromotors extremely attractive biosensing tools.

\section{Acknowledgement}

This work was supported by the Defense Threat Reduction Agency Joint Science and Technology Office for Chemical and Biological Defense (Grants nos. HDTRA1-13-1-0002 and HDTRA1-14-10064), and by the European Union's Horizon 2020 Research and Innovation programme under the Marie Sklodowska-Curie Grant agreement no. 656687.

\section{Appendix A. Supplementary material}

Supplementary data associated with this article can be found in the online version at doi:10.1016/j.talanta.2017.02.068.

\section{References}

[1] J. Wang, Nanomachines: Fundamentals and Applications, Wiley-VCH, Weinheim, Germany, 2013.

[2] S. Sánchez, L. Soler, J. Katuri, Chemically powered micro-and nanomotors, Angew. Chem. Int. Ed. 54 (5) (2015) 1414-1444.

[3] M. Guix, C.C. Mayorga-Martinez, A. Merkoçi, Nano/micromotors in (bio) chemical science applications, Chem. Rev. 114 (12) (2014) 6285-6322.

[4] H. Wang, M. Pumera, Fabrication of micro/nanoscale motors, Chem. Rev. 115 (16) (2015) 8704-8735.

[5] X. Yu, Y. Li, J. Wu, H. Ju, Motor-based autonomous microsensor for motion and counting immunoassay of cancer biomarker, Anal. Chem. 86 (9) (2014) 4501-4507.

[6] J. Orozco, S. Campuzano, D. Kagan, M. Zhou, W. Gao, J. Wang, Dynamic isolation and unloading of target proteins by aptamer-modified microtransporters, Anal. Chem. 83 (20) (2011) 7962-7969.

[7] D. Kagan, S. Campuzano, S. Balasubramanian, F. Kuralay, G.-U. Flechsig, J. Wang, Functionalized micromachines for selective and rapid isolation of nucleic acid targets from complex samples, Nano Lett. 11 (5) (2011) 2083-2087.

[8] S. Balasubramanian, D. Kagan, C.M. Jack Hu, S. Campuzano, M.J. Lobo-Castañon, N. Lim, D.Y. Kang, M. Zimmerman, L. Zhang, J. Wang, Micromachine-enabled capture and isolation of cancer cells in complex media, Angew. Chem. Int. Ed. 50 (18) (2011) 4161-4164

[9] S. Campuzano, J. Orozco, D. Kagan, M. Guix, W. Gao, S. Sattayasamitsathit, J.C. Claussen, A. Merkoçi, J. Wang, Bacterial isolation by lectin-modified microengines, Nano Lett. 12 (1) (2012) 396-401.

[10] D. Vilela, J. Orozco, G. Cheng, S. Sattayasamitsathit, M. Galarnyk, C. Kan, J. Wang, A. Escarpa, Multiplexed immunoassay based on micromotors and microscale tags, Lab Chip 14 (18) (2014) 3505-3509.

[11] M. García, J. Orozco, M. Guix, W. Gao, S. Sattayasamitsathit, A. Escarpa, A. Merkoçi, J. Wang, Micromotor-based lab-on-chip immunoassays, Nanoscale 5 (4) (2013) 1325-1331.

[12] J. Wang, Self-propelled affinity biosensors: moving the receptor around the sample, Biosens. Bioelectron. 76 (2016) 234-242.

[13] J. Orozco, B. Jurado-Sánchez, G. Wagner, W. Gao, R. Vazquez-Duhalt, S. Sattayasamitsathit, M. Galarnyk, A. Cortés, D. Saintillan, J. Wang, Bubblepropelled micromotors for enhanced transport of passive tracers, Langmuir 30 (18) (2014) 5082-5087.

[14] E. Morales-Narváez, M. Guix, M. Medina-Sánchez, C.C. Mayorga-Martinez, A. Merkoçi, Micromotor enhanced microarray technology for protein detection, Small 10 (13) (2014) 2542-2548.

[15] T. Ito, N. Aoki, S. Kaneko, K. Suzuki, Highly sensitive and rapid sequential cortisol detection using twin sensor QCM, Anal. Methods 6 (18) (2014) 7469-7474.

[16] P. Batista, A. Pereira, Biomarkers in neurodegenerative diseases: cortisol, J. Mol. Biomark. Diagn. 7 (277) (2016) 2.

[17] B. Sun, Y. Gou, Y. Ma, X. Zheng, R. Bai, A.A.A. Abdelmoaty, F. Hu, Investigate electrochemical immunosensor of cortisol based on gold nanoparticles/magnetic functionalized reduced graphene oxide, Biosens. Bioelectron. 88 (2017) 55-62.

[18] R.M. Sapolsky, Why stress is bad for your brain, Science 273 (5276) (1996) $749-751$.

[19] E.K. Do Yup Lee, M.H. Choi, Technical and clinical aspects of cortisol as a 
biochemical marker of chronic stress, BMB Rep. 48 (4) (2015) 209.

[20] N. Murase, S.-i. Taniguchi, E. Takano, Y. Kitayama, T. Takeuchi, A molecularly imprinted nanocavity-based fluorescence polarization assay platform for cortisol sensing, J. Mater. Chem. B 4 (10) (2016) 1770-1777.

[21] D. Appel, R.D. Schmid, C.-A. Dragan, M. Bureik, V.B. Urlacher, A fluorimetric assay for cortisol, Anal. Bioanal. Chem. 383 (2) (2005) 182-186.

[22] T. Sink, R. Lochmann, K. Fecteau, Validation, use, and disadvantages of enzymelinked immunosorbent assay kits for detection of cortisol in channel catfish, largemouth bass, red pacu, and golden shiners, Fish Physiol. Biochem. 34 (1) (2008) 95-101.

[23] J. Fang, J.C. Zhou, E.H. Lan, B. Dunn, J.I. Zink, Bio-hybrid materials for immunoassay-based sensing of cortisol, J. Sol-Gel Sci. Technol. 50 (2) (2009) $176-183$.

[24] S.K. Pasha, A. Kaushik, A. Vasudev, S.A. Snipes, S. Bhansali, Electrochemical immunosensing of saliva cortisol, J. Electrochem. Soc. 161 (2) (2014) B3077-B3082.

[25] M. Moreno-Guzmán, M. Eguílaz, S. Campuzano, A. González-Cortés, P. YáñezSedeño, J.M. Pingarrón, Disposable immunosensor for cortisol using functionalized magnetic particles, Analyst 135 (8) (2010) 1926-1933.

[26] A. Kaushik, A. Yndart, R.D. Jayant, V. Sagar, V. Atluri, S. Bhansali, M. Nair, Electrochemical sensing method for point-of-care cortisol detection in human immunodeficiency virus-infected patients, Int. J. Nanomed. 10 (2015) 677.

[27] A. Kaushik, A. Vasudev, S.K. Arya, S.K. Pasha, S. Bhansali, Recent advances in cortisol sensing technologies for point-of-care application, Biosens. Bioelectron. 53 (2014) 499-512.

[28] S. Campuzano, P. Yánez-Sedeño, J.M. Pingarrón, Electrochemical bioaffinity sensors for salivary biomarkers detection, TrAC Trends Anal. Chem. 86 (2017) $14-24$ 